\title{
Neural processing of biomedical data for improving driving safety
}

\author{
F. Barcellona ${ }^{1}$, F. Filippi ${ }^{1}$, M. Panella ${ }^{2}$, A. M. Bersani ${ }^{3}$ \\ \& A. Alessandrini ${ }^{1}$. \\ ${ }^{I}$ D.I.T.S. Department, University of Rome "La Sapienza", Italy \\ ${ }^{2}$ INFO-COM Department, University of Rome "La Sapienza", Italy \\ ${ }^{3}$ Me.Mo.Mat Department, University of Rome "La Sapienza", Italy
}

\begin{abstract}
Driving ability can be impaired by fatigue, drowsiness, drugs and alcohol, all of which have been implicated in causing road traffic accidents. Acute hypoglycaemia, which is the most common side effect of insulin therapy in individuals with diabetes, may also compromise driving skills. Other than by forbidding people to drive, the potential danger can be reduced by monitoring health and consciousness of drivers, by providing them with feedback on their conditions using, eventually, an emergency centre or biofeedback. In this paper, we propose the use of a signal processing system based on neural networks for system modelling and prediction. In particular, using neural networks we will reproduce the glucose temporal evolution without invasive technique for drivers, with the aim of preventing loss of consciousness while driving and hence improving road safety. Some illustrative trials will be shown in this regard. This research work is supported by the "CTL Excellence Centre (Centro di Ricerca sul Trasporto e la Logistica)" co-funded by the Italian Ministry of University, Education and Research and by the University of Rome "La Sapienza".

Keywords: modelling and prediction, diabetes, data acquisition and analysis, neural network, road safety.
\end{abstract}

\section{Introduction}

Driving is an activity that demands complex psychomotor skills, good visuospatial functions, rapid information processing, vigilance, and satisfactory judgment. Hypoglycaemia is a common side-effect of insulin therapy for 
diabetes, which may be associated with significant morbidity and can interfere with everyday activities such as driving. Medical studies have demonstrated that cognitive functions which are critical to driving (such as visual information processing, attention, reaction times and hand-eye coordination) are impaired by some critical biological parameters such as hypoglycaemia, high body temperature or low skin conductance level. In fact, clinical studies have shown that driving performance is adversely affected by these biological alterations [2]. Hypoglycaemia is recognized to cause some crashes and consequently, careful avoidance of hypoglycaemia is recommended before and during driving. This is particularly relevant for drivers receiving intensive insulin therapy, who may experience hypoglycaemia more frequently. We propose in this paper a signal processing system based on neural networks by which we will reproduce the glucose temporal evolution without invasive technique for drivers. The aim is to prevent loss of consciousness while driving and hence to improve the road safety. The neural computing system is implemented on a board unit mounted on the vehicle; such a unit is capable of collecting, storing, elaborating and communicating data obtained in real-time from the vehicle and its components (i.e., driver, cargo, surrounding environment, etc.).

\section{Diabetes mellitus and driving performance}

Glucose is the most important physiological stimulation for insulin secretion. Insulin reply to a protracted glucose stimulation of the $\beta$-cells is split into two phases: a first, high, secretion, rapidly decreasing, and a second, delayed, secretion peak, during all the stimulation period. When glucose is no more able to stimulate the $\beta$-cells, in the human subject several dysfunctions appear, among which one of the most serious is the so-called "diabetes mellitus" (DM). It is characterized by hyperglycaemia, due to a complete absence of insulin or to a partial deficit, related to its reduced biological efficiency. DM can be classified into two forms [5]:

a) DM type 1, insulin-dependent (IDMM); it is characterized by a quite complete absence of insulin secretion; it represents the $10-15 \%$ of all the DM pathologies;

b) DM type 2, insulin-independent (NIDMM); it is characterized by a low insulin secretion, associated to tissue refractoriness to insulin activity; it represents the $85-90 \%$ of all the DM pathologies.

One acquired complication of insulin therapy, which increases in prevalence with duration of diabetes, is the impaired awareness of hypoglycaemia. Acute hypoglycaemia, the most common side effect of insulin therapy, may also compromise driving skills. Although its development does not invariably result in revocation of the driving license, it is considered to be a major risk for driving by most licensing authorities. Impaired awareness of hypoglycaemia is associated with more profound cognitive dysfunction, which takes longer to 
recover after acute hypoglycaemia than is experienced by individuals with normal awareness. Studies during hypoglycaemia have demonstrated that cognitive function does not return to a normal state until 40-90 minutes after normoglycemia has been restored. Functions that are most affected by hypoglycaemia include rapid decision-making, sustained attention, analysis of complex visual stimuli, "mental flexibility," memory of recently learned information, and hand-eye coordination. In addition, hypoglycaemia diminishes the speed of visual information processing, affects contrast sensitivity and promotes mood changes, including increased irritability and anger, which may be relevant to road safety.

\section{Prediction of glucose behaviour}

The performance of a predictor depends on how accurate it models the unknown context delivering the sequence to be predicted. Due to the actual importance of forecasting, the technical literature is plenty of methods for implementing a predictor, especially in the field of neural networks $[6,7]$. All of them only partially match the context, also in the favorable case of signals not contaminated by the presence of spurious components; consequently, a prediction error inevitably arises.

Given a sampled sequence $s(n)$, the general approach to its prediction at distance $m, m>0$, consists in expressing the sample $s(n+m)$ in function of the previous samples $s(k), k \leq n$. The latter are usually grouped into a vector $\underline{\mathrm{X}}_{\mathrm{n}} \in \mathfrak{R}^{\mathrm{N}}$, where integer $\mathrm{N}$ represents in this case the number of samples in $\underline{x}_{n}$ and it is also known as the order of the predictor. Based on this approach, two problems must be solved: the determination of the most suitable set $\underline{x}_{n}$ of previous samples; the determination of the function $s(n+m)=f^{(m)}\left(x_{n}\right)$, $f^{(m)}: \Re^{\mathrm{N}} \rightarrow \mathfrak{R}$, relating future samples to the previous ones. Such a function is usually approximated by means of a synthetic model $\widetilde{f}^{(m)}(\underline{x})$. Early methods proposed were based on the estimation of linear models. For example, the simplest prediction approach is the one concerning linear autoregressive (AR) models, where each input vector $\underline{x}_{n}$ is constituted by $\mathrm{N}$ consecutive samples of $s(n)$ and the target output is the sample $s(n+m)$ to be predicted; i.e.:

$$
\begin{aligned}
& \underline{x}_{n}=\left[\begin{array}{lll}
s(n) s(n-1) & \ldots s(n-N+1)
\end{array}\right] \\
& \tilde{f}_{A R}^{(m)}(\cdot)=-\sum_{i=1}^{N} a_{i} x_{n i} \Rightarrow \tilde{s}(n+m)=-\sum_{i=1}^{N} a_{i} s(n-i+1)
\end{aligned}
$$

The coefficients $a_{i}, i=1 \ldots N$, of the function $\widetilde{f}_{A R}^{(m)}(\cdot)$ can be determined in this case by relying on global statistical properties of the sequence $s(n)$ as, for example, its autocorrelation function. 
The way to determine the input vectors $\underline{x}_{n}$, based on past samples of $s(n)$, is called embedding technique. Based on well-known techniques for time series analysis [8], a useful expression for the input vector is $\underline{x}_{n}=\left[\begin{array}{lllll}s(n) & s(n-T) & s(n-2 T) & \ldots & s(n-(N-1) T)\end{array}\right]$, where $N$ and $T$ are also referred to as embedding dimension and time lag, respectively. Although such parameters can be estimated by analyzing the time series, the resulting values for $N$ and $T$ right not be feasible for glucose prediction while driving. According to clinical experience and some preliminary trials, a suited choice, which we will use in the following, is $N=5$ and $T=1$ using a twenty minutes sampling rate. Thus, on the basis of the previous approach, the basic problem to be solved in a prediction task is the approximation of an unknown function from the observation of a limited number of input-output pairs, i.e. the embedded vectors $\underline{x}_{n}$ and target outputs that must be predicted. Therefore, the approximation is achieved through a regression function, which is learnt by using a training set of samples with the aim of maximizing its generalization capability. The latter, in this case, is the capability to accurately predict a set of successive samples of the glucose sequence, which can be estimated over a test set of samples not used during training. The approximation problem is generally referred to as statistical regression or function estimation. Neural networks are also regression models, which are particularly suited in this regard. Usually, function estimation methods are based on a parametric basis function expansion, i.e.:

$$
\tilde{f}(\underline{x})=\sum_{j=1}^{C} a_{j} B_{j}\left(\underline{x}, \underline{\theta}_{j}\right)
$$

where the expansion coefficients $\mathrm{a}_{\mathrm{j}}$ and the basis function parameters $\underline{\theta}_{\mathrm{j}}, \mathrm{j}=1 \ldots \mathrm{C}$, are determined during the learning procedure by using the training set. An important classification of function estimation methods based on neural networks is [9]: $k$-Nearest Neighbors ( $k$-NN), Projection Pursuit Regression (PPR), Multivariate Adaptive Regression Splines (MARS), Multi-Layer Perceptron (MLP), Constrained Topological Mapping (CTM), Radial Basis Function (RBF) networks, Adaptive Neuro-Fuzzy Inference Systems (ANFIS) and Mixture of Gaussian (MoG) density model.

Among all the possible alternatives that can be used for this aim, a promising and well-known solution in the technical literature is represented by neural networks based on a MoG density models. In fact, the effectiveness of MoG neural networks in approximating real-world data sequences has been already established in $[4,10,11]$.

In order to train an MoG network we can adopt the SHEM (Splitting Hierarchical Expectation Maximization) algorithm [9]. It is based on the maximum likelihood approach for estimating the parameters of the whole Gaussian mixture, i.e. the $\underline{\theta}_{j}$ parameters of each Gaussian Kernel $B_{j}$. The most important benefit of SHEM is the automatic selection of the number $\mathrm{C}$ of Gaussian components. 


\section{Analysis of results}

In the following we show the results with a comparison between classical linear model (AR) and some different neural networks (MoG, RBF, ANFIS). As a comparison index, we will use the Signal to-Noise ratio (SNR) defined by

$$
10 \log _{10} \frac{\sum_{n} s_{n}^{2}}{\sum_{n}\left(s_{n}-\widetilde{s}_{n}\right)^{2}}
$$

where $\widetilde{s}_{n}$ represent the predicted value and $s_{n}$ the actual value. We analyzed different cases using the predictors previously mentioned, by illustrating some different figures that represent the predicted glucose sequence in different cases. We have used a training set of 500 elements representing 10 different time series each composed by 50 samples. As test set we used 150 elements representing 10 different time series each composed by 15 sequences.

Table 1: $\quad$ Comparison between Signal-to-Noise Ratio (SNR).

\begin{tabular}{|l|l|l|l|}
\hline Predictors & SNR-Test 1 & SNR-Test 2 & SNR-Test3 \\
\hline MoG & $22.195 \mathrm{~dB}$ & $33.456 \mathrm{~dB}$ & $37.343 \mathrm{~dB}$ \\
\hline RBF & $21.817 \mathrm{~dB}$ & $29.95 \mathrm{~dB}$ & $34.325 \mathrm{~dB}$ \\
\hline ANFIS & $19.292 \mathrm{~dB}$ & $25.727 \mathrm{~dB}$ & $29.321 \mathrm{~dB}$ \\
\hline LINEAR & $2.845 \mathrm{~dB}$ & $3.8742 \mathrm{~dB}$ & $5.4587 \mathrm{~dB}$ \\
\hline
\end{tabular}

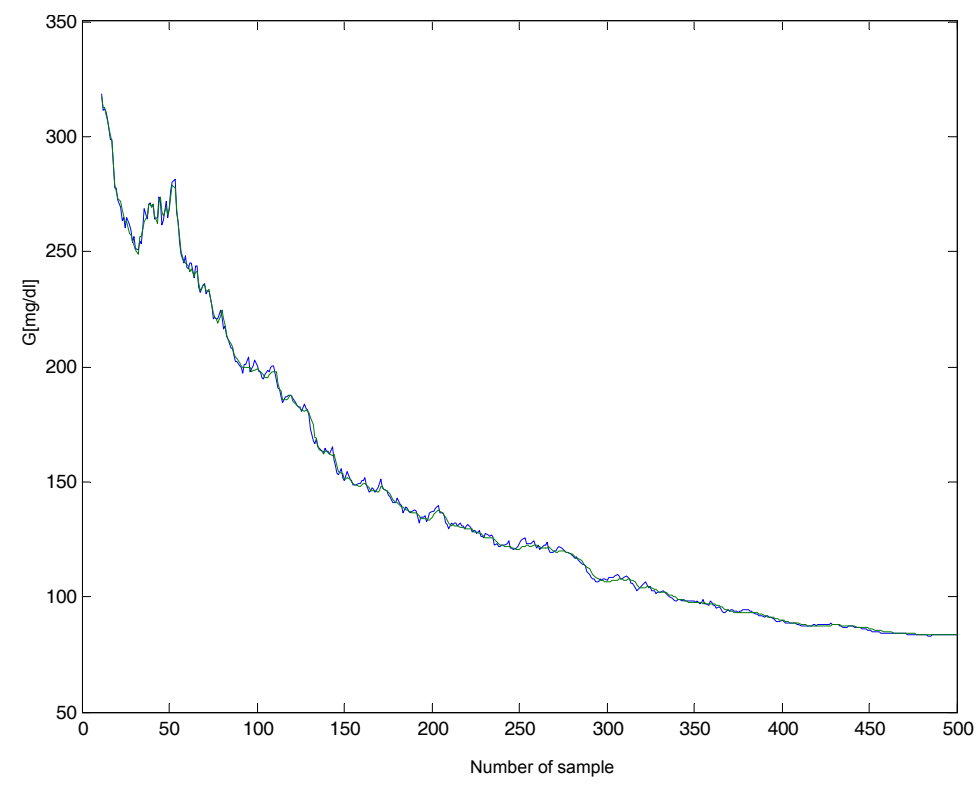

Figure 1: Analysis of MoG predictor. 
218 Modelling in Medicine and Biology VI

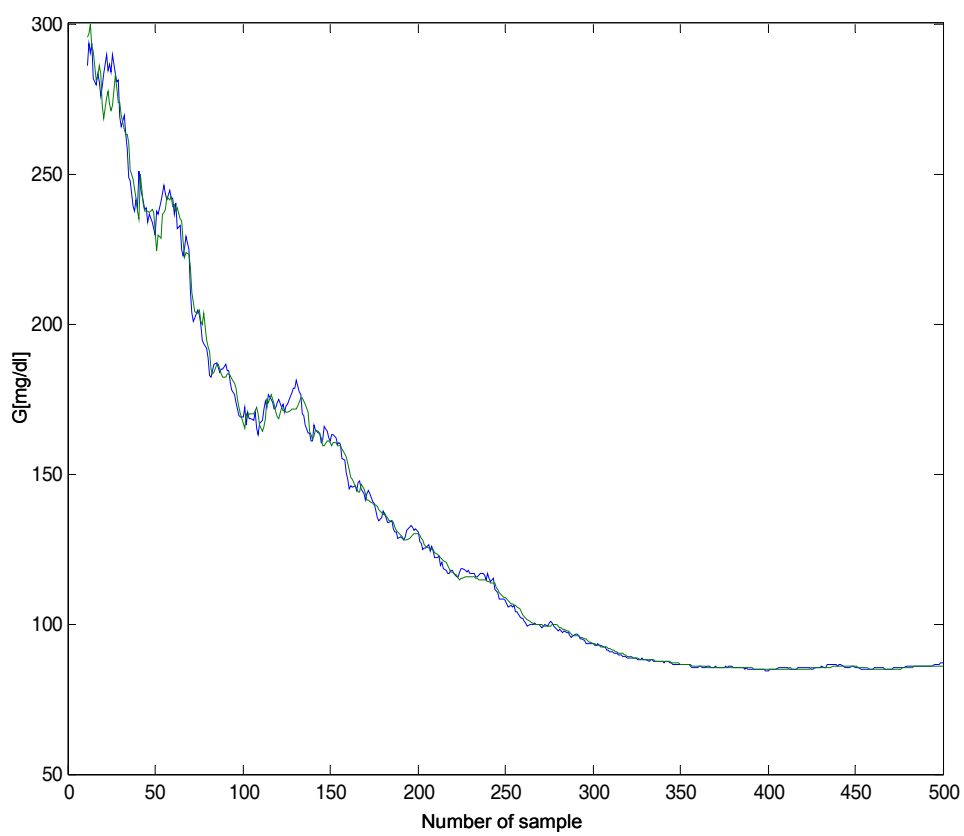

Figure 2: $\quad$ Analysis of RBF predictor.

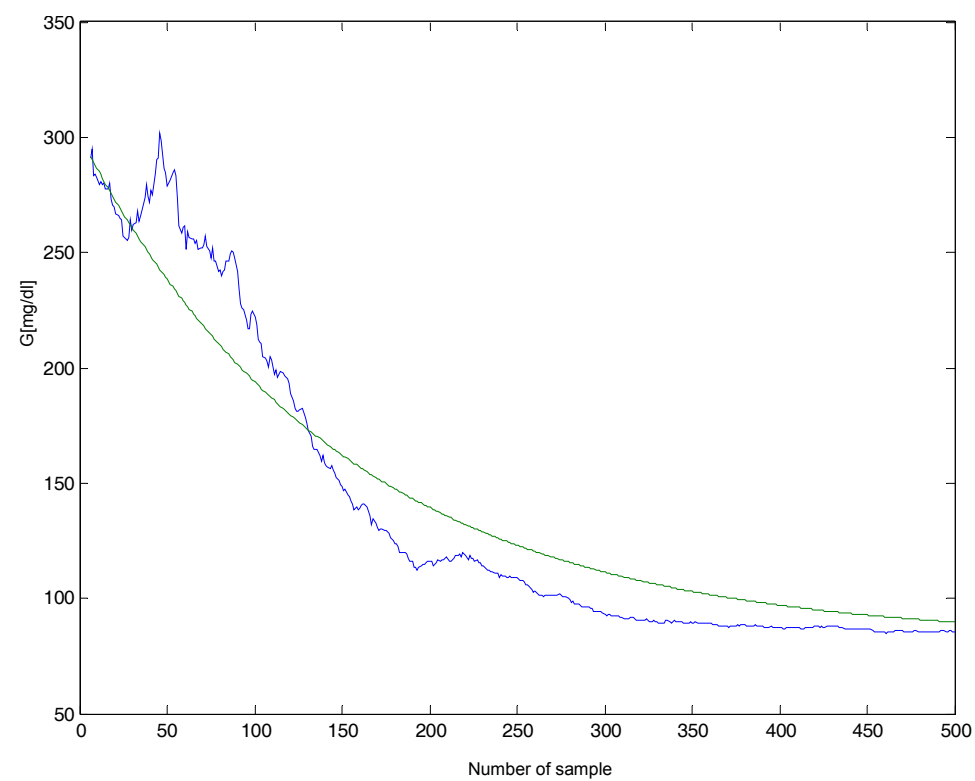

Figure 3: $\quad$ Analysis of linear model. 
We show in fig. 1 the glucose temporal evolution predicted by MoG neural network, in fig. 2 the temporal glucose evolution predicted by RBF neural network and in fig. 3 the temporal glucose behaviour predicted by linear autoregressive model (AR).

\section{Conclusion}

In this paper, we have observed that neural networks are suitable for modelling the temporal glucose behaviour, and more satisfactory than the linear autoregressive model. Consequently, from a safety and clinical point of view, this method may have some interesting implications, in order to single out possible pathological cases, without involving techniques which are invasive for drivers, with the aim to prevent loss of consciousness while driving and hence to improve the road safety. At present time, we are evaluating the performances of training and testing on real board units mounted on some prototype vehicles.

\section{References}

[1] Deary I.J., "Effects of hypoglycaemia on cognitive function", in Hypoglycaemia and Diabetes: clinical and Physiological aspects, pp. 80-92, Frier B.M., Fisher B.M., Eds., London, Edward Arnold, 1993.

[2] Frier B.M., "Driving and Diabetes", BMJ 305, pp. 1238-1239, 1992.

[3] Palmieri B., Taggi F., "Safety, Wellness, Fitness \& Check Driver: stato dell'arte e proposte innovative per la sicurezza di guida automobilistica", available at 'http://www.iss.it/sitp/sicu/publ/0003/0041.pdf', (in Italian).

[4] Panella M., Barcellona F., Bersani A.M. "Neural network in modeling glucose-insulin behaviour", Lecture Notes in Computer Science, SpringerVerlag, 2004, (in press).

[5] Faglia, "Malattie del sistema endocrino e del metabolismo", 1997.

[6] A.S. Weigend and N.A. Gershenfeld, "Time Series Prediction: Forecasting the Future and Understanding the Past", Addison-Wesley, 1994.

[7] H. White, "Learning in artificial neural networks: a statistical perspective", Neural Computation 1, 425-464, 1989.

[8] Abarbanel H.D.I, "Analysis of observed chaotic data", Springer-New York, 1986.

[9] M. Panella, A. Rizzi, G. Martinelli. "Refining accuracy of environmental data prediction by mog neural network". Neurocomputing (55),521-549. 2003.

[10] M. Panella, A. Rizzi, F.M. Frattale Mascioli, and G. Martinelli, "Improved Time Series Forecasting by a Twofold Neural Predictor", in: Proc. of EANN 2001, 196-203 (Cagliari, Italy, 2001).

[11] M. Panella, A. Rizzi, F.M. Frattale Mascioli, and G. Martinelli, "Constructive MoG Neural Networks for Pollution Data Forecasting", in: Proc. of IJCNN, 417-422 (Honolulu, Hawaii, U.S.A., 2002). 